\title{
Research on Joint Handoff Algorithm in Vehicles Networks
}

\author{
Yuming Bi, Lei Tian, Mengmeng Liu, Zhenzi Liu, and Wei Chen \\ Key Laboratory of Universal Wireless Communications, Ministry of Education Beijing University of Posts and Telecommunications, \\ Mailbox No. 92, Beijing 100876, China \\ Correspondence should be addressed to Yuming Bi; biyuming10507@sina.com
}

Received 1 October 2015; Revised 12 January 2016; Accepted 21 March 2016

Academic Editor: Sujit Dey

Copyright (C) 2016 Yuming Bi et al. This is an open access article distributed under the Creative Commons Attribution License, which permits unrestricted use, distribution, and reproduction in any medium, provided the original work is properly cited.

With the communication services evolution from the fourth generation $(4 \mathrm{G})$ to the fifth generation (5G), we are going to face diverse challenges from the new network systems. On the one hand, seamless handoff is expected to integrate universal access among various network mechanisms. On the other hand, a variety of $5 \mathrm{G}$ technologies will complement each other to provide ubiquitous high speed wireless connectivity. Because the current wireless network cannot support the handoff among Wireless Access for Vehicular Environment (WAVE), WiMAX, and LTE flexibly, the paper provides an advanced handoff algorithm to solve this problem. Firstly, the received signal strength is classified, and the vehicle speed and data rate under different channel conditions are optimized. Then, the optimal network is selected for handoff. Simulation results show that the proposed algorithm can well adapt to high speed environment, guarantee flexible and reasonable vehicles access to a variety of networks, and prevent ping-pong handoff and link access failure effectively.

\section{Introduction}

With the continuous development of the fifth generation (5G) mobile communications and the ever-maturing standards, intelligent terminals and mobile Internet and networking systems have shown a trend of rapid development worldwide. Meanwhile, the vehicular communication system has a wide range of engineering application values in the future communication markets. Therefore, strong research activities have been stimulated in the field of Wireless Access for Vehicular Environment (WAVE). However, due to the high mobility of vehicles, the seamless handoff becomes a new bottleneck in vehicular communication system. Several literatures have investigated the handoff problem. Reference [1] proposed a handoff method based on the detection of received signal strength (RSS), while [2] mainly focused on the traditional data rate. In [3], the packet loss rate was considered to decide handoff. In [1-3], certain physical quantities, also called detection values, are utilized to judge whether the handoff should be done. Besides, the cost functions can also be used to make handoff decisions, such as throughput and quality of service (QoS) [4-6]. Some scholars believed that multiple factors should be considered. Thus, several handoff algorithms based on multiple attribute decision (MAD) appeared. The operator policies, terminal properties, customer performance, and the application QoS level are all taken into account $[7,8]$. In addition, [9] used an analytic hierarchy process (AHP) to acquire the various performance parameters to make a judgment for the weights of the network. Reference [10] utilized the remaining bandwidth of vertical handoff algorithm for switching among heterogeneous networks, relying on the QoS Basic Service Set (QBSS) with limitation. All of the above-mentioned handoff algorithms aimed at cellular communication systems. There is still lack of research on handoff problem in vehicular communication system. Different from the traditional cellular networks which are based on fixed base stations (BSs) and mobile cellular networks, the vehicular communication systems require high speed mobile terminals (MTs) to use self-organizing network (SoN) or cooperation to realize endto-end (e2e) data interaction directly [10]. As a consequence, the vehicular communication system may easily produce call dropping and ping-pong effects. In addition, some papers focused on the vertical handoff and the others only took the horizontal handoff into consideration. But it is clear that the future wireless access network will consist of wireless 
networks with various services and coverage ranges. So as to accurately obtain the "always best connect" performance networks, the joint vertical and horizontal handoff in heterogeneous networks will become an increasingly important research hotspot, although little researche has been done on it. In order to allow users to roam among various systems transparently and seamlessly, this paper presents a novel algorithm which not only supports the horizontal handoff in the same network but also can classify the RSS and optimize velocity speed and data rate under different channel conditions to choose an optimal network to handoff. For the sake of low computational complexity, RSS is adopted to handle horizontal handoff and the fuzzy logic decision algorithm is used to address vertical handoff. There is no doubt that the fuzzy logic decision algorithm takes many features of the mobile terminals (MTs) and access network into consideration and thus makes the handoff algorithm easier for hardware implementation. The remainder of this paper is organized as follows. Section 2 describes the background of handoff process. Section 3 indicates the proposed handoff algorithm and gives the related channel model. Numerical results are presented in Section 4. Finally, Section 5 concludes the whole paper.

\section{Background}

2.1. The Brief of Handoff. It is not difficult to find that the $5 \mathrm{G}$ wireless access network will consist of access networks with various coverage and data rates. The hybrid network system can take advantage of each system to provide high QoS to users. According to the relationship between the source and target network, the handoff process can be divided into horizontal handoff and vertical handoff, as shown in Figure 1. In general, horizontal handoff refers to handoff in the same access technology, while vertical handoff is the handoff process occurring between different access network technologies. No matter which handoff process happens, the ultimate objective is to keep "always best connect" (ABC) for users [11-13]. That is to say, when a mobile user connects to hybrid access networks concurrently, all they will do is to choose a best adjacent cell in the same network or access network for its service requirement purpose. In mobile communication systems, there exists a certain overlap area between adjacent cells, called handoff belt. When the mobile terminal moves into this area, the wireless link handoff process must be conducted effectively. However, in the hybrid network systems, the coverage area of several networks may be overlapped. Thus, a new handoff scenario in the hybrid network system has to occur in the near future [14]. In order to minimize the link dropping probability, the horizontal and vertical handoff should be considered jointly.

2.2. The Network Architecture and Protocol. The existing network architecture consists of mobile terminal, access network, and core network. In the access network, the base station (eNodeB) bears the control function of wireless system and makes the data transmission rate become faster. Moreover, the boundaries between access network and core network are blurred. The mobile terminal is connected to the

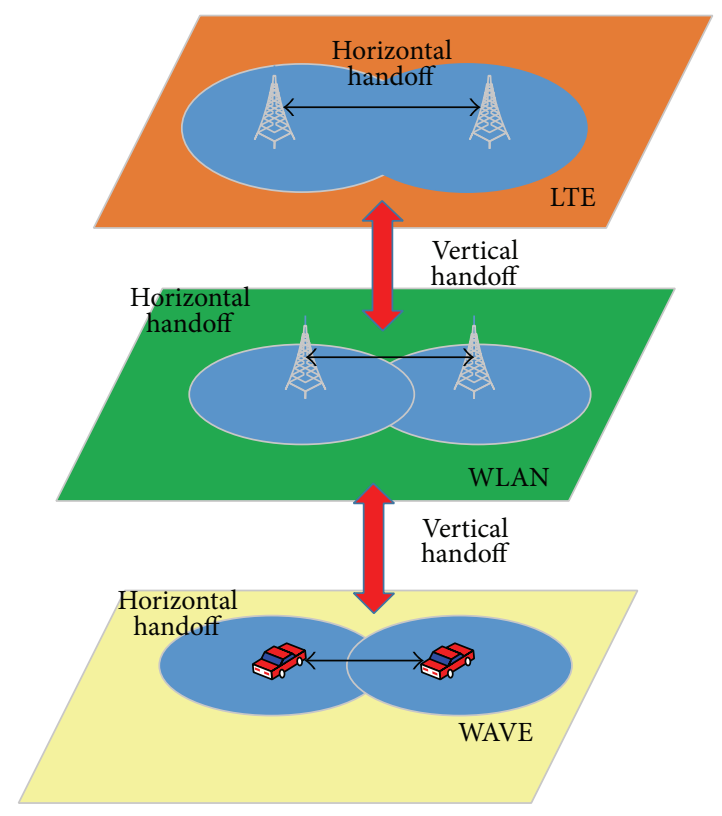

FIGURE 1: The schematic map of handoff.

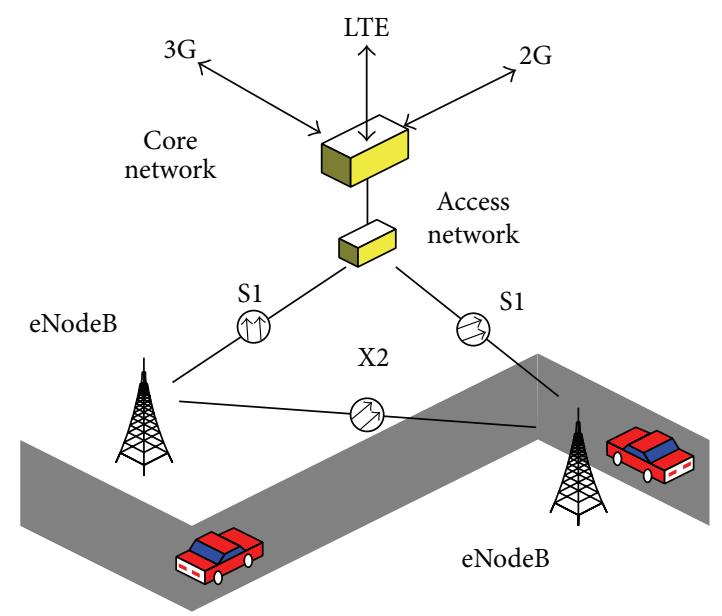

FIgURE 2: The vehicular wireless communication system.

access network via air interface. The eNodeB uses $\mathrm{S} 1$ interface to connect to the core network and X2 interface to link with another eNodeB, respectively, as shown in Figure 2.

The typical vehicular communication system is divided into three subsystems: core network, vehicle, and ground parts. The vehicular subsystem mainly includes the vehicular information platform, the communication module, the system multiplexing module, the $2 \mathrm{G} / 3 \mathrm{G}$ automotive micro base station unit, and the $\mathrm{Wi}$-Fi hotspots equipment. It always directly communicates with the ground stations through standard onboard processing unit which is responsible for the large capacity data transmission. The ground subsystem mainly includes the eNodeB radio remote unit (RRU) and the S1 and X2 interface optic fibers. This paper assumes that the parts of the core network are related to the access network. Specifically, the core network uploads the users 
service information and the whole system control information. Moreover, the access network is utilized for converting the format of $2 \mathrm{G} / 3 \mathrm{G}$ and separating them into the different service networks.

Therefore, as discussed above, there are two types of handoff process; one is based on X2 interface switch and the other is connected with S1 interface. For the horizontal handoff, the data will be passed through X2 interface between eNodeBs. When the user equipment (UE) needs vertical handoff, the source eNodeB will trigger the S1 interface to switch information from eNodeB to access network and core network.

2.3. The Procedure of Handoff. The handoff process has three steps. (1) Measurement of handoff: at first, UE always tries to find out whether there is a new available network or not. In the vertical handoff process, many conditions of switch are considered, such as signal receiving intensity, user preference, network cost, and load balance. The common handoff decision will be triggered when network congestion is serious or current network signal and QoS are weak. The handoff may also be held when the current network cannot provide satisfactory businesses to the user. (2) Switching decision stage: there are three kinds of control modes to make the handoff decision: network control, terminal control, and terminal auxiliary switch. The system will synthetically take into account the situation of each alternative network, the terminal characteristics, and the user's preferences for the current business. In some special circumstances, other factors (such as QoS and system performance security) will also be considered. (3) The execution phase of handoff: this stage is completing the handoff process and switching the communication services from the current access point to the target network. To ensure the completion of the handoff, some protocols are needed; more details about it can be found in [15].

\section{The Handoff Algorithm}

In this section, we present a novel scheme based on the joint RSS and fuzzy logic handoff algorithm. Figure 3 shows the algorithm flow chart. Assume that the WLAN system has successfully established the connection. The vehicle measurement equipment always detects the various access network signals. Once the RSS is higher than that from other access networks, the station keeps the data connection with WLAN and meanwhile rises the horizontal handoff mode. Otherwise, the mobile station waits for the optimal network selection time before comparing the RSS again. After the RSS comparison, if the RSS in WLAN is still higher than the others, the whole handoff process returns to the initial phase, and the vehicle measurement equipment retests the various access network signals. Otherwise, the algorithm mechanism will decide to start the vertical handoff mode which makes decision based on fuzzy inference engine by utilizing Zadeh rules, and the details are described as follows.

3.1. The Algorithm of Horizontal Handoff. During each period, the UE in either idle or connection state measures

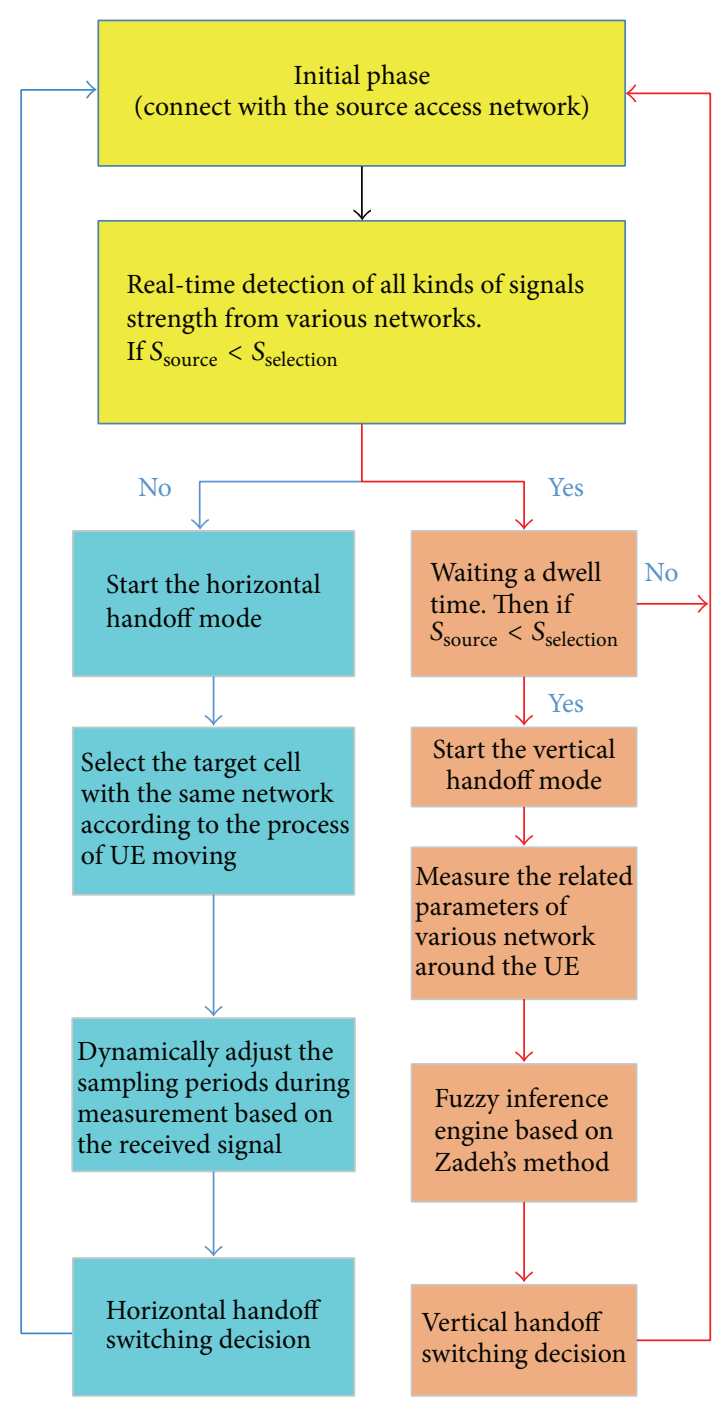

FIgURE 3: The flow chart of handoff algorithm.

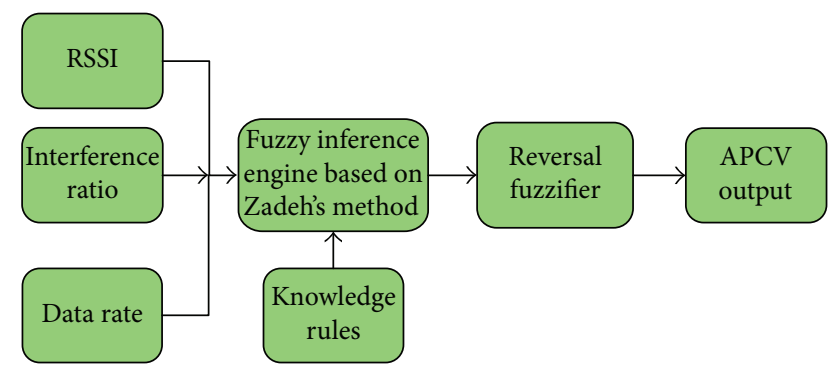

FIgURE 4: The fuzzy logic decision scheme.

the related power parameters related to both the source cell and the target one. The measurement parameters include reference signal received power (RSRP), reference signal received quality (RSRQ), and reference signal strength indicator (RSSI). The RSRP is the received power value of the pilot signal from base station. It is calculated by the difference 
between the reference signal which transmits from the base station and the path loss:

$$
\mathrm{RSRP}=P_{T x}-\mathrm{PL}(\mathrm{dB}),
$$

where $P_{T x}$ is the transmitter power of the pilot signal and PL represents the path loss. The RSRQ can be calculated as

$$
\mathrm{RSRQ}=N * \frac{\mathrm{RSRP}}{\mathrm{RSSI}},
$$

where $N$ is the number of Resource Blocks (RB) in terms of bandwidth. The RSSI is the measurement value of the received carrier's power in the whole system bandwidth; however the carrier sends not only data and control information but also some interference and noise information. In order to make the handoff decision reasonable, the acquired information from the measurement data needs to be submitted to the Radio Resource Control (RRC) layer. In addition, the measured reference signal should be smoothed by filters before being transmitted to the RRC for eliminating random fluctuations [16]. The trigger condition of handoff execution is as follows: the QoS of target cell is better than the currently serving cell and the difference exceeds a specified threshold when the duration is greater than the trigger delay time. The judgment criteria are shown as

$$
P_{\text {target }}>P_{\text {source }}+\mathrm{O}_{f}+H,
$$

where $P_{\text {target }}$ and $P_{\text {source }}$ represent the strength power of the target cell and source cell, respectively, and $O_{f}$ means the offset between related source cell and target cell. When the measurement value of the received signal is consistent with (3), the timer will start working. If the relationship described by (3) continuity satisfies the rule of time to trigger (TTT), the system will determine to drive the handoff process execution. The decision threshold $H$ and TTT have great influence on the performance of the algorithm. Larger $H$ and longer delay time of departure will make the handoff more difficult. Therefore, as a result of switch, it is easy to lead to link connection fail, namely, drop link. If $H$ is too small it will cause frequent switching, namely, ping-pong phenomenon.

3.2. The Algorithm of Vertical Handoff. In this part, we will focus on how to use the algorithm for a vertical operation based on fuzzy logic. As we all know, the general elements of collection set for the membership can only take 0 and 1 . In 1965 , L. A. Zadeh expanded the membership from only two values ( 0 and 1$)$ to any values within 0 and 1 , which was a sign that the membership function with fuzzy sets could be represented by fuzzy probability. The fuzzy subset $B$, which belongs to the theory domain $T$, is a function characterized by a collection of membership and can be mapped as follows:

$$
\mu_{B}: T \longrightarrow[0,1],
$$

where $\mu_{B}$ means the membership function of the fuzzy subset. $\mu_{B}(t)$ represents the extent to which the elements $t$ in the theory domain $T$ belong to fuzzy subset $B$. The larger value of it the higher probability of belonging to $B$. For a given theory

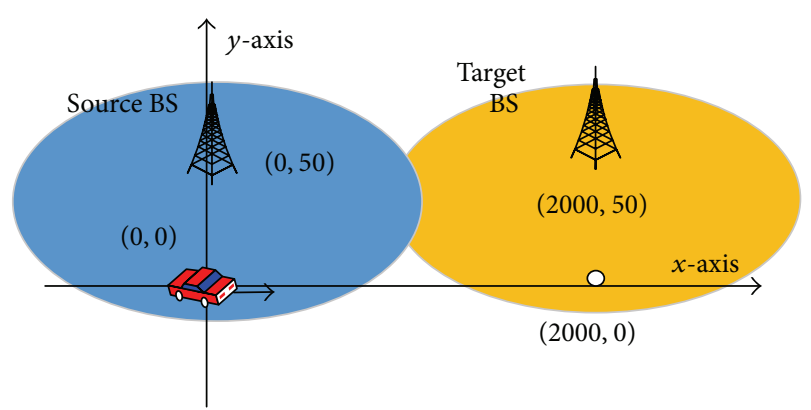

FIGURE 5: The horizontal handoff simulation model.

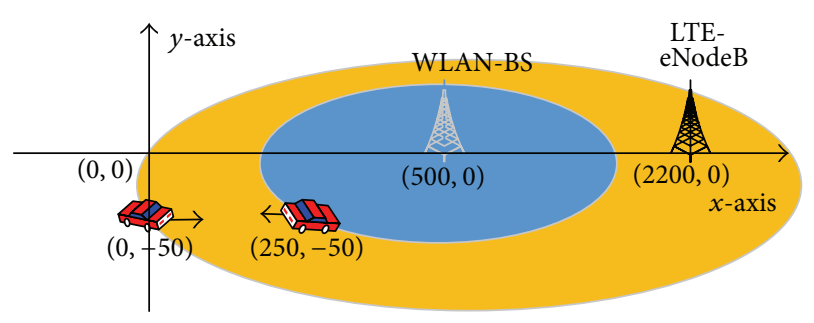

FIgURE 6: The vertical handoff simulation model.

domain of $E$, a kind of word which is related to $E$ constitutes a set $P$. Its semantics is represented by the function $R$ which maps the relationship between set $P$ and $E$. Therefore the word set is a fuzzy function and it can be described as

$$
R(a, e)=\mu_{B}(e),
$$

where $e$ is the element of $E$. And the membership function $\mu_{B}(a, e)$ means the extended relationship between $a$ (which belongs to the set $P$ ) and $e$ (which belongs to the theory domain $E$ ):

$$
\mu_{R}: P \times E \longrightarrow[0,1]
$$

In order to deduce the fuzzy logic relationship, we will introduce the principle of Zadeh's method: if the fuzzy relationship "If $A$ then $B$ " can be represented by " $A \rightarrow B$," where $A \in U, B \in V$, then the fuzzy logic relationship $R(u, v)$ is defined as

$$
\text { Zadeh: } R(u, v)=(A(u) \wedge B(v)) \vee(1-A(u)),
$$

where " $\wedge$ " and " $\vee$ " stand for the supremum and infimum operator with logic relationship, respectively. For a given $A^{*} \epsilon$ $U$, if the relationship matrix $R$ is known, then $B^{*} \in V$ can be calculated by

$$
B^{*}=A^{*} \circ R,
$$

where "o" means the supremum operator with logic relationship.

The proposed fuzzy logic decision strategy consists of three steps (as shown in Figure 4): fuzzy inference engine based on Zadeh's method, reversal fuzzifier, and APCV output. At first, the input parameters such as RSSI, IR, and DR are mapped into inference engine by utilizing knowledge 


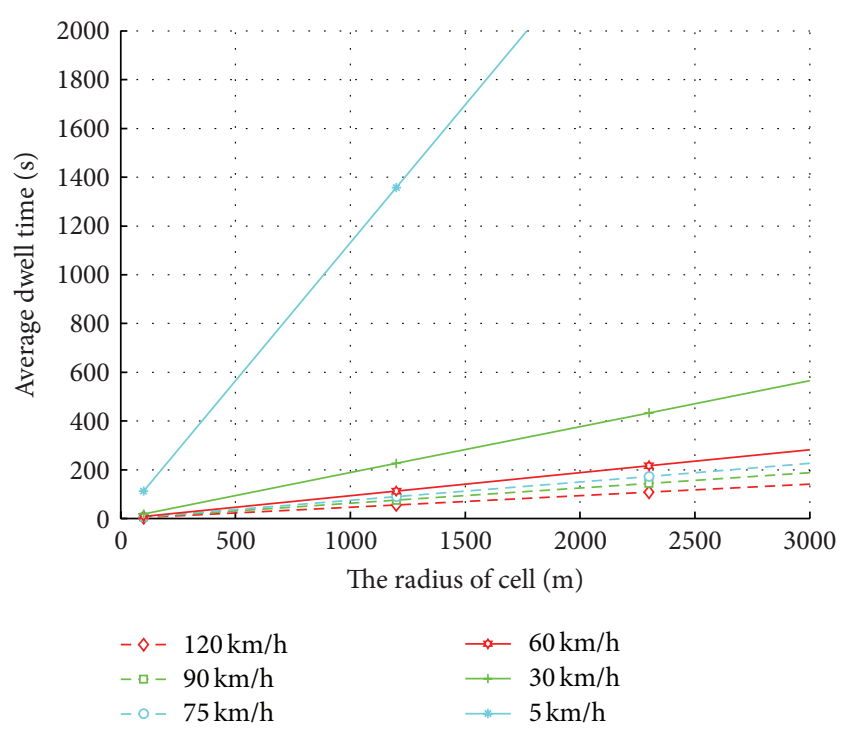

(a)

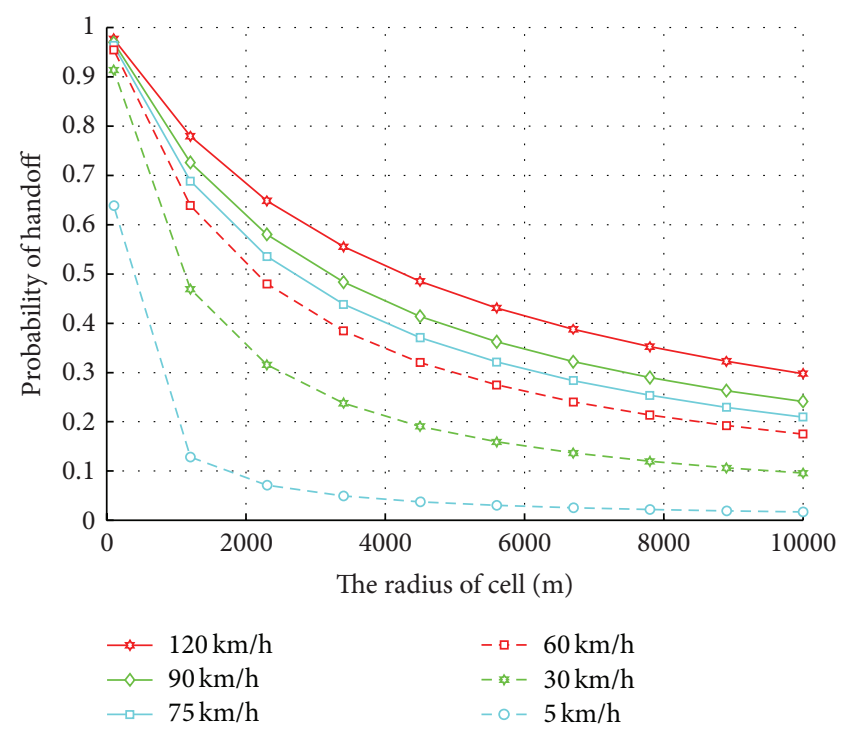

(b)

FIGURE 7: The handoff probability and the average time with various cell radius and user speeds.

rules. And then the inference engine will make a fuzzy decision according to the mapping values. But as mentioned above the output of the fuzzy inference module is still a fuzzy set and has no effects on the controlled object directly. Therefore, a "translation" process is needed to transfer a fuzzy value to a precise one $f_{0}$. This step is called "reversal fuzzifier." Through comparison with this precise value, we can get the access point candidacy value (APCV) output. The goal of this "reversal fuzzifier" is to find the maximum membership degree function. The "reversal fuzzifier" selects the element which will make the value of membership function the highest as the output signal. And the rules could be described as

$$
\mu\left(f_{0}\right) \geq \mu(f), \quad f \in F .
$$

In case of nonunique maximum value of the membership function, the average value will be taken as follows:

$$
f_{0}=\frac{1}{N} \sum_{i=1}^{N} f_{i}, \quad \mu\left(f_{i}\right) \geq \mu(f) .
$$

In different networks, different fuzzy inference models are adopted. The membership function is designed to ensure comparability among different networks at the same time. At last, in order to complete the behavior of handoff, the system will select the highest APCV network to access. There are three parameters that need to be detected in the fuzzy module: RSSI, data rate, and interference ratio. Gaussian and $S$-scheme distribution are chosen as the fuzzy membership because of their great performance with respect to real-time control.

Figure 9 shows the WLAN membership functions. For example, RSSI stands for the received signal strength indicator. Therefore we make such an assumption to describe its knowledge rules (as shown in Figure 9(c)): (i) the membership function is $S$-scheme distribution when RSSI is in a given interval from $-95 \mathrm{dBm}$ to $-80 \mathrm{dBm}$; (ii) if RSSI belongs to the interval [ $-90 \mathrm{dBm},-70 \mathrm{dBm}$ ], then the membership function follows Gaussian distribution; and (iii) once the value of RSSI is higher than $-80 \mathrm{dBm}$, the membership function is $S$-scheme distribution as well. The fuzzy set of RSSI, the set of data rate, and the fuzzy set of interference ratio in WLAN are [good, normal, weak] with value set $[3,2,1]$. Hence, the output fuzzy sets are mapped as

$$
S=\sum_{i=1}^{3} M_{i} k
$$

Take a regular WLAN network as an example: if RSSI is weak and data rate is weak and interference ratio is weak, then $S$ is 3. For the WLAN network, each input has three fuzzy sequences, so there are 27 fuzzy rules in the knowledge rules. Due to the sensitivity to UE speed in V2V network, 5 fuzzy variable sequences are used (45 fuzzy rules) in the knowledge rules. The input parameters in the fuzzy logic module are mapped into different fuzzy sets, and the output would be acquired utilizing the maximum membership degree function method. The fuzzy logic outputs decision value indicates that the stable degree of difference between two network decisions is lower than the threshold level; thus the connection with the source network cell will be held on. Otherwise, the handoff execution will be done if the hysteresis time is longer than a certain value; that is,

\section{VALUE_TARGET - VALUE_SOURCE $\geq$ Hysteresis,}

VALUE_SOURCE $\leq$ Threshold. 


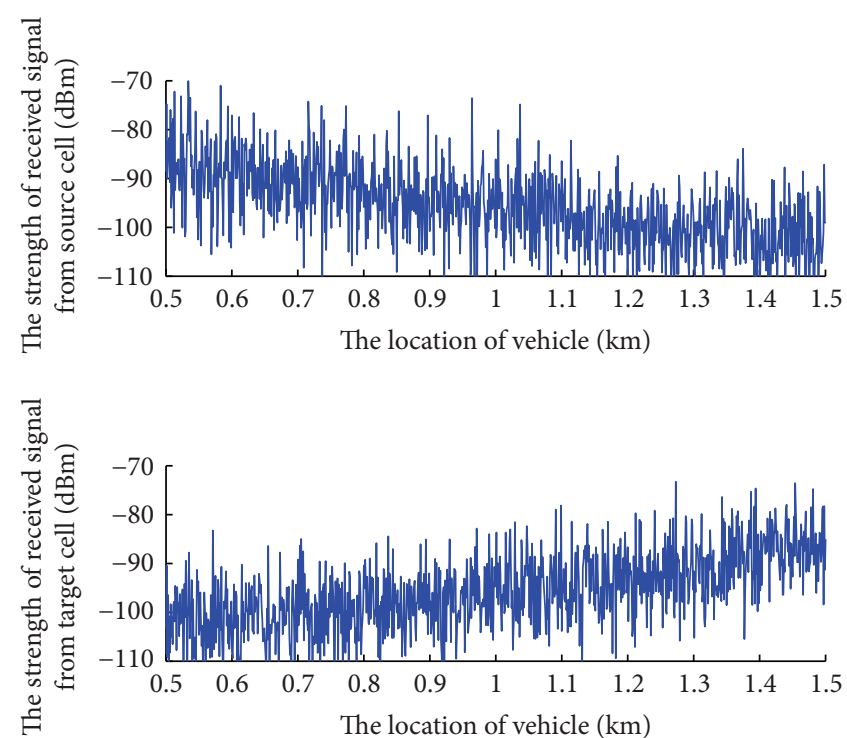

(a) The strength of received signal from source and target cell

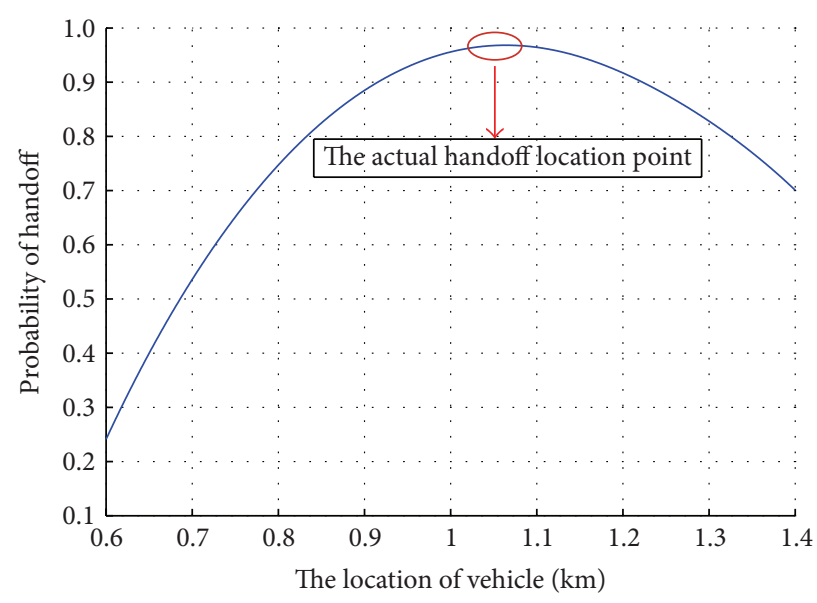

(c) The actual handoff location point

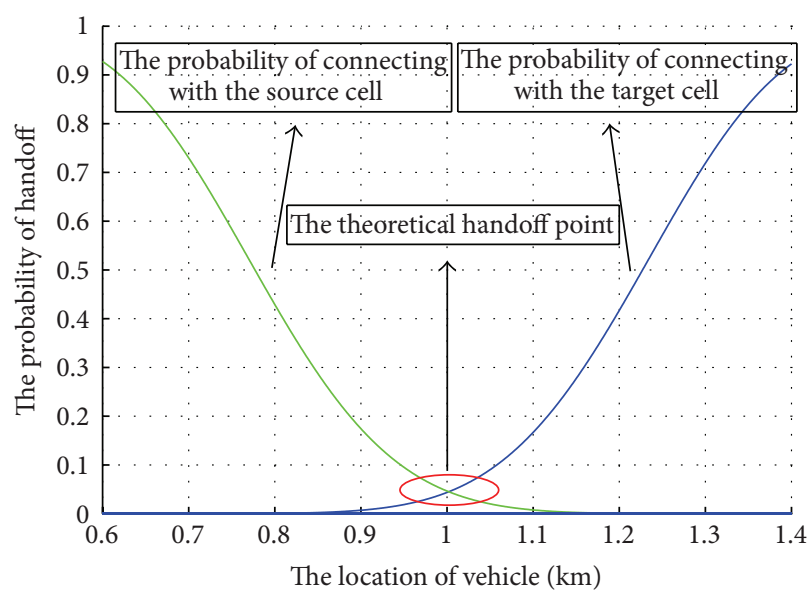

(b) The probability of handoff

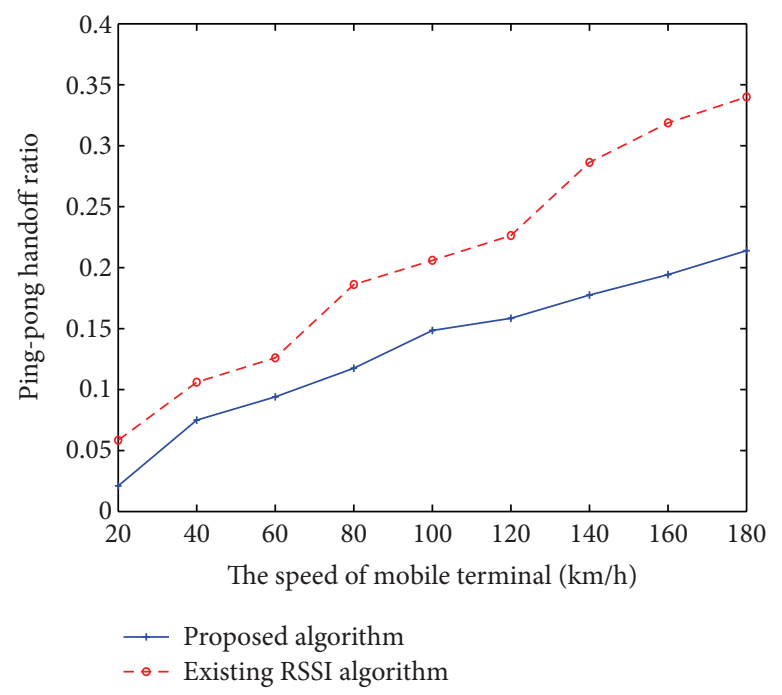

(d) The performance comparison

FIGURE 8: The horizontal handoff simulation results.

3.3. Channel Model. This subsection will adopt the COST 231 Hata channel model to realize the real-time simulation [17, 18], which is depicted as

$$
\begin{aligned}
\mathrm{PL}(\mathrm{dB})= & \left(39.7-7.01 \log _{10}\left(h_{\mathrm{BS}}\right)\right) \log _{10}\left(\frac{d}{1000}\right) \\
& +42.9+\left(36.54-1.2 h_{\mathrm{MS}}\right) \log _{10}\left(f_{c}\right) \\
& -12.98 \log _{10}\left(h_{\mathrm{BS}}\right)+0.74 h_{\mathrm{MS}}+C
\end{aligned}
$$

where $h_{\mathrm{BS}}$ and $h_{\mathrm{MS}}$ represent the height of BS and MS, respectively, $d$ means the distance between BS and MS, $f c$ denotes the center frequency, and $C$ is a constant.
The channel impulse response (CIR) from transmitter antenna element $s$, to receiver element $u$, for cluster $n$ is expressed as

$$
\begin{aligned}
& h_{u, s, n}(t)=\sqrt{\frac{P_{n} \sigma_{\mathrm{SF}}}{M}} \sum_{m=1}^{M}\left(\sqrt{G_{B S}\left(\theta_{n, m, \mathrm{AoD}}\right)}\right. \\
& \cdot \exp \left(j\left[k d_{s} \sin \left(\theta_{n, m, \mathrm{AoD}}\right)+\phi_{n, m}\right]\right) \\
& \cdot \sqrt{G_{\mathrm{MS}}\left(\theta_{n, m, \mathrm{AoA}}\right)} \times \exp \left(j k d_{u} \sin \left(\theta_{n, m, \mathrm{AoA}}\right)\right) \\
& \left.\quad \times \exp \left(j k\|v\| \cos \left(\theta_{n, m, \mathrm{AoA}}-\theta_{v}\right) t\right)\right),
\end{aligned}
$$

where $G_{\mathrm{BS}}$ and $G_{\mathrm{MS}}$ are the antenna gain for BS and MS, respectively, $d_{s}$ and $d_{u}$ are the uniform distances (m) between transmitter elements and receiver elements, respectively, $k$ 


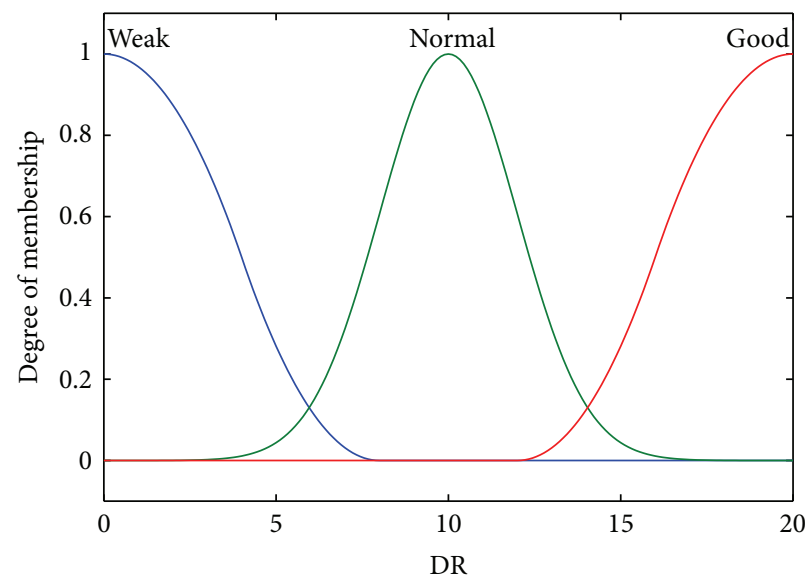

(a) Data rate

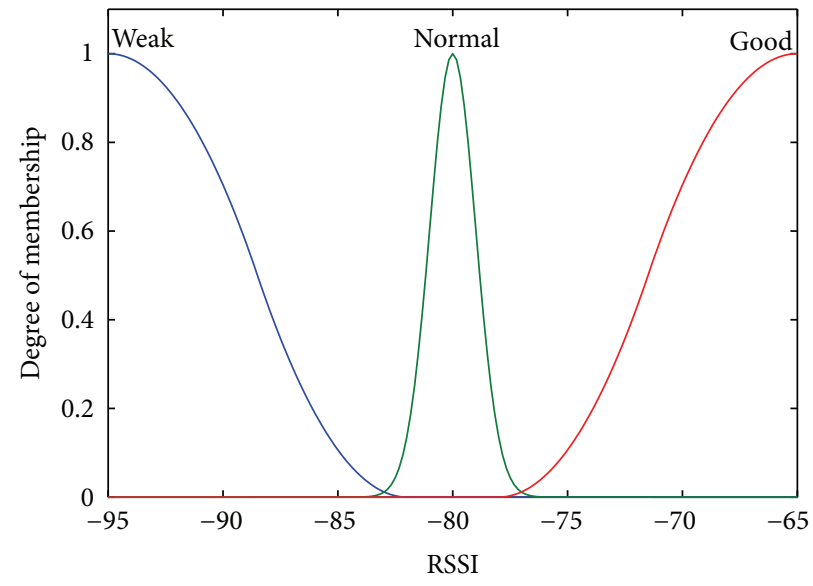

(c) RSSI

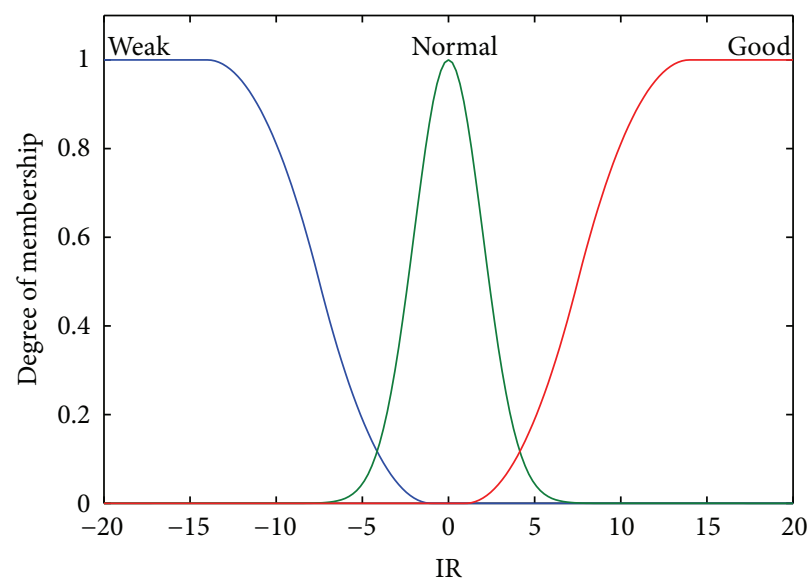

(b) Interference ratio

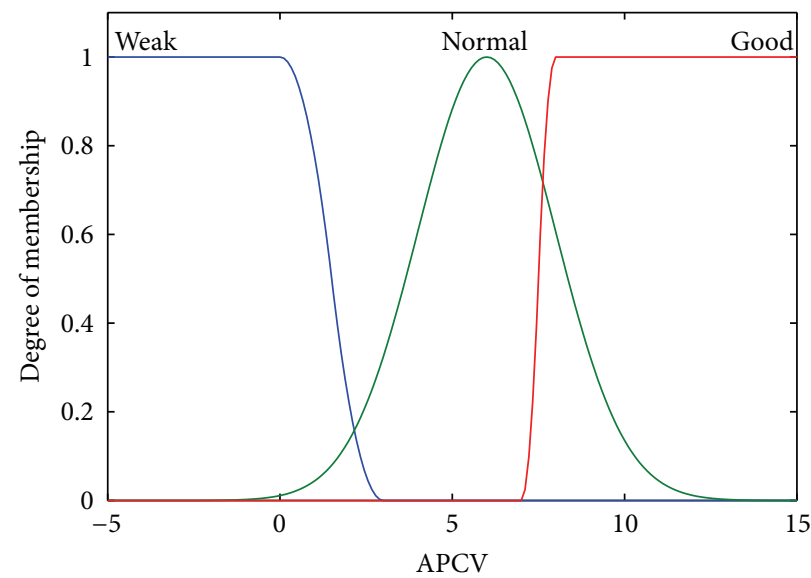

(d) APCV

FIgURE 9: The value of fuzzy set.

is the cross polarization power ratio in linear scale, $v$ is the moving speed of user, and $\theta_{n, m, \mathrm{AoA}}$ and $\theta_{n, m, \mathrm{AoD}}$ mean the angle of arrival (AoA) and angle of departure (AoD) with the $m$ th subpath in $n$th path, respectively. Assume that there are 6 paths and each path includes 20 subpaths.

\section{The Simulation and Validation}

To evaluate the realistic performance of our proposed algorithm, the simulation model, scenarios, and various parameters are developed using MATLAB. The performance of horizontal and vertical handoff algorithms is tested separately.

Figure 7 compares the handoff probability and average dwell time with different moving speed and cellular radius. It is found that the expansion of cellular radius and decreasing vehicle moving speed will lead to increase of average dwell time and channel holding time. In particular, the relationship between the coverage radius and dwell time is obviously linear in the low vehicle speed regions. On the contrary, with the increase of vehicle speed, the handoff probability will continue to decrease.

The horizontal handoff is simulated using the following parameters (as shown in Figure 5): the point coordinates of source BS and target BS are $[0,50]$ and $[2000,50]$, which means that the distance between the source BS and target BS is $2000 \mathrm{~m}$ and the distance between road and BS is $50 \mathrm{~m}$; the vehicle speed is $80 \mathrm{~km} / \mathrm{h}$ and is moving from $[0,0]$ to [2000,0]; the heights of BS and MS are $30 \mathrm{~m}$ and $1 \mathrm{~m}$ separately; the transmitted power is $44 \mathrm{dBm}$ and the standard deviation of channel shadow fading is $8 \mathrm{~dB}$.

Figure 8 shows the simulation results of horizontal handoff. It is clearly seen that when the vehicle moves from $[500,0]$ to $[1500,0]$, the strength of received signal from source BS is reducing while the signal level from target BS is growing. Meanwhile, the handoff probability shows a similar trend, and the handoff should occur at the points near $[1000,0]$ according to the theoretical judgment of holding probability. It can also be observed that the handoff happens at the peak of the curve; namely, the coordinate of the vehicle is $[1055,0]$. There is a little far distance from the actual handoff location to the theoretical one due to the hysteresis effect of dwell time on the joint handoff algorithm. As we all know, the existing RSSI algorithm depends on the decisions of priority on the mobile node. In particular, the priority is divided into low priority and high priority. The low priority which is below certain level estimate receives signal strength from 


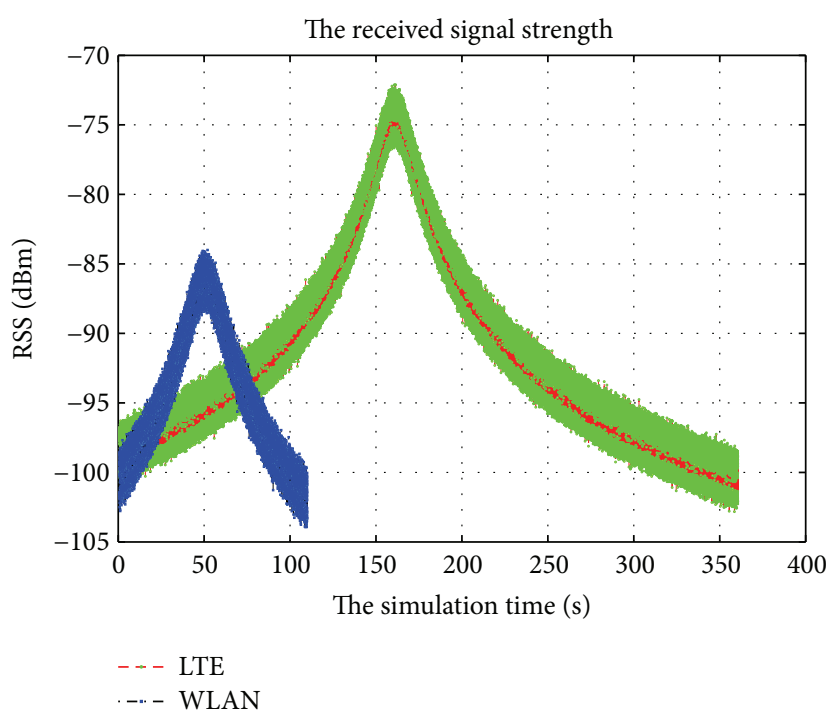

(a) RSS

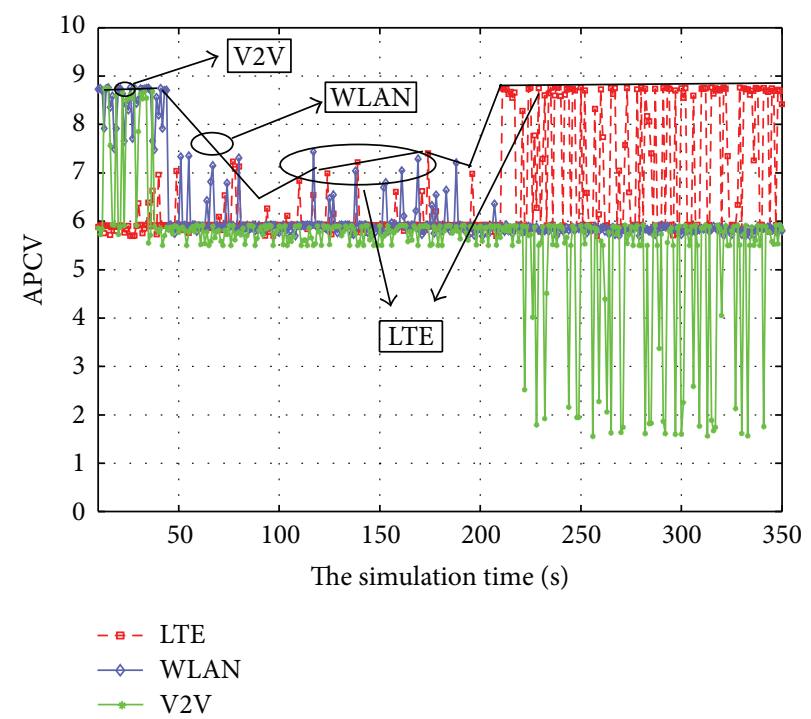

(c) APCV and state of handoff

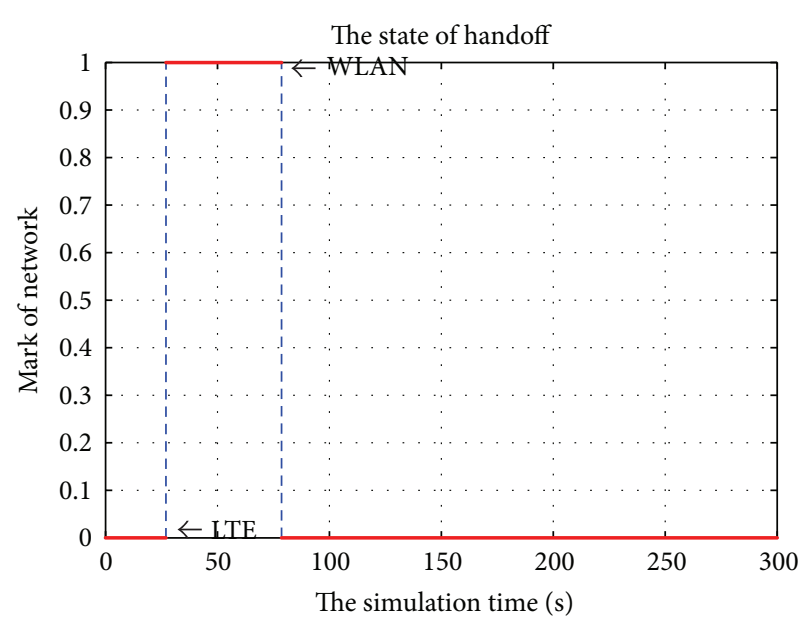

(b) The state of handoff

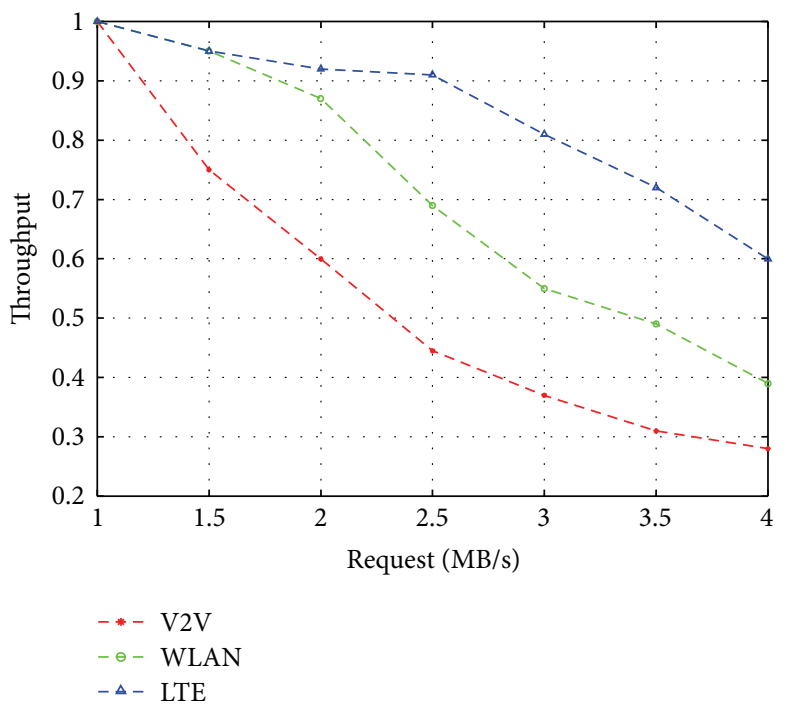

(d) Throughput versus request

FIgURE 10: The vertical handoff simulation results.

another cell before it starts handoff in the cell. Therefore, Figure 8 (d) presents a performance comparison between the existing RSSI horizontal handoff algorithm and our proposed schemes. It is clear that, no matter how much the speed of users is, the ping-pong handoff ratio of existing RSSI horizontal handoff algorithm is higher than the proposed schemes. It proves that ping-pong effect can be avoided effectively by using dwell timer and hysteresis parameters.

The vertical handoff simulation is divided into two parts: one focuses on testing whether the handoff could be applied automatically and another part analyzes the performance of the proposed algorithm. Firstly, assume a car is moving from the initial points $[0,-50]$ to $[2500,-50]$ at the speed of $45 \mathrm{~km} / \mathrm{h}$, and the coordinates of WLAN-BS and LTE-eNode are $[500,0]$ and $[2200,0]$, respectively (as shown in Figure 6).
Figure 10(a) shows the alternative trend of the RSS during the whole movement and Figure 10(b) shows the actual handoff results. It is not difficult to find such a handoff process: the car accessed LTE at first and then changed the network to WLAN because it ran close to the WLAN-BS; when it left the coverage of WLAN, the handoff happened again to replace WLAN by LTE signals. Obviously, because of different path loss and shadow fading due to different center frequency, the vehicle utilizes LTE network to communicate and only connects to WLAN network when the mobile terminal is very close to the BS of WLAN. Secondly at the performance simulation parts, similar to the functional simulation, the coordinates of the car and the BS of WLAN and LTE network are the same as the former ones. The biggest difference setting between the function and the performance simulation part 


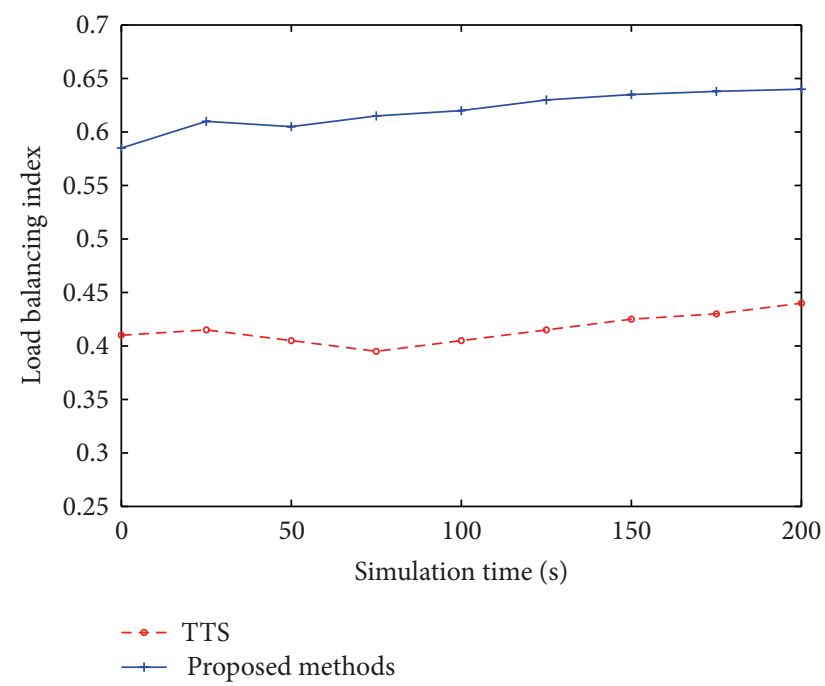

(a)

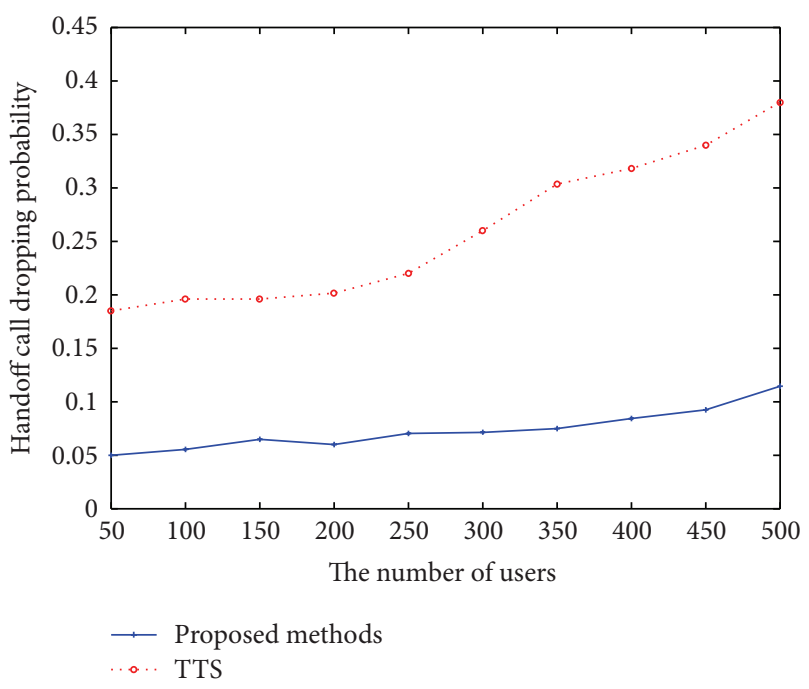

(b)

FIGURE 11: The performance comparison in different methods.

is that it is assumed that another car is moving from points $[250,-50]$ to points $[-250,-50]$ at the same speed with the target car.

As it is observed in Figure 10(c), at the beginning, the car utilized V2V network to communicate, and after then the handoff process changes the access network from WLAN to LTE according to the rules for choosing the highest value of APCV (as shown by black lines) among them as access network. The handoff process is consistent with the previous simulation except that it accessed V2V network initially. By comparing the throughput of different networks (as shown in Figure 10(d)), it could be found that the network performance of LTE is the best, then there is WLAN, and V2V is the worst. From the aspects of vertical handoff, the triangle and trapezoid shapes (TTS) are always chosen as fuzzy membership functions in many engineering applications. Moreover, the load balancing degree and handoff call dropping probability are used to evaluate the performance of handoff. Therefore, after comparison from Figure 11(a), we can find that the load balancing degree of our proposed schemes is higher than the TTS. In addition, as illustrated in Figure 11(b), call dropping probability is lower in our proposed schemes than that of TTS, especially in the larger number of users conditions. In general, the simulation results validate that our proposed scheme outperforms the existing vertical handoff algorithm.

\section{Conclusion}

This paper proposed a dynamic handoff algorithm in vehicles networks to solve the conflicts between BS and MS, such as call blocking, call dropping, and ping-pong phenomenon. Simulation results validate that the presented joint handoff algorithm can effectively address the handoff problem among various access networks by taking into account different scenarios, different vehicle speeds, and channel conditions.

\section{Competing Interests}

The authors declare that they have no competing interests.

\section{Acknowledgments}

This research is supported in part by China Important National Science and Technology Specific Projects (no. 2013ZX03001020-002) and by National Key Technology Research and Development Program of China (no. 2012BAF14B01) and by National Natural Science Foundation of China (no. 61171105 and no. 61322110) and by 863 Program Project (no. 2015AA01A703) and Doctor Funding Program (no. 201300051100013).

\section{References}

[1] F. Siddiqui and S. Zeadally, "Mobility management across hybrid wireless networks: trends and challenges," IEEE Computer Communications, vol. 11, no. 5, pp. 45-54, 2005.

[2] N. Nasser, A. Hasswa, and H. Hassanein, "Handoffs in fourth generation heterogeneous networks," IEEE Communications Magazine, vol. 44, no. 10, pp. 96-103, 2006.

[3] M. Ylianttila, J. Makeela, and P. Mahonen, "Vehicular telematics over heterogeneous wireless networks: a survey," Computer Communications, vol. 33, no. 7, pp. 775-793, 2010.

[4] X. Liu, L.-G. Jiang, and C. He, "Vertical handoff algorithm based on fuzzy logic in aid of pre-decision method," Acta Electronica Sinica, vol. 35, no. 10, pp. 1989-1993, 2007.

[5] Y. B. Kang, K. Xu, Y. L. Shen et al., "Optimal distributed vertical handoff strategies in vehicular heterogeneous networks," IEEE Journal on Communications, supplement 1, pp. 69-73, 2009.

[6] D. Ma and M. Ma, "A QoS-based vertical handoff scheme for interworking of WLAN and WiMAX," in Proceedings of the IEEE Global Telecommunications Conference (GLOBECOM '09), pp. 1-6, Honolulu, Hawaii, USA, December 2009.

[7] M. Liu, Z.-C. Li, X.-B. Guo, and K. Zheng, "A movement trend based self-adaptive vertical handoff algorithm and its performance evaluation," Chinese Journal of Computers, vol. 31, no. 1, pp. 112-119, 2008.

[8] Y. Li, M. Li, B. Cao, Y. Wang, and W. Liu, "Dynamic optimization of handover parameters adjustment for conflict avoidance 
in long term evolution," China Communications, vol. 10, no. 1, pp. 56-71, 2013.

[9] H. Hu, J. Zhang, X. Zheng, Y. Yang, and P. Wu, "Selfconfiguration and self-optimization for LTE networks," IEEE Communications Magazine, vol. 48, no. 2, pp. 94-100, 2010.

[10] W. Tian, Y. Zhao, Y. Zhong, M. Xu, and C. Jing, "Dynamic and integrated load-balancing scheduling algorithm for cloud data centers," China Communications, vol. 8, no. 6, pp. 117-126, 2011.

[11] K. Shafiee, A. Attar, and V. C. M. Leung, "Optimal distributed vertical handoff strategies in vehicular heterogeneous networks," IEEE Journal on Selected Areas in Communications, vol. 29, no. 3, pp. 534-544, 2011.

[12] J. Zhang, H. C. B. Chan, and V. C. M. Leung, "A locationbased vertical handoff decision algorithm for heterogeneous mobile networks," in Proceedings of the IEEE Global Telecommunications Conference (GLOBECOM '06), pp. 1-5, San Francisco, Calif, USA, December 2006.

[13] S. Lee, K. Sriram, K. Kim, Y. H. Kim, and N. Golmie, "Vertical handoff decision algorithms for providing optimized performance in heterogeneous wireless networks," IEEE Transactions on Vehicular Technology, vol. 58, no. 2, pp. 865-881, 2009.

[14] H. Zhai, X. Chen, and Y. Fang, "How well can the IEEE 802.11 wireless LAN support quality of service?" IEEE Transactions on Wireless Communications, vol. 4, no. 6, pp. 3084-3094, 2005.

[15] L.-D. Chou, J.-M. Chen, H.-S. Kao, S.-F. Wu, and W. Lai, "Seamless streaming media for heterogeneous mobile networks," Mobile Networks and Applications, vol. 11, no. 6, pp. 873-887, 2006.

[16] Y. Sun, F. Yang, and Z. Shi, "Distributed network management system with load balancing," IEEE Journal on Communications, vol. 30, no. 3, pp. 34-41, 2009.

[17] Z.-Y. Feng, P. Zhang, and Y.-J. Zhang, "A joint load control algorithm with terminal selection for the reconfigurable systems," Journal of Electronics and Information Technology, vol. 31, no. 4, pp. 893-896, 2009.

[18] X. Yan, N. Mani, and Y. A. Sekercioglu, "A traveling distance prediction based method to minimize unnecessary handovers from cellular networks to WLANs," IEEE Communications Letters, vol. 12, no. 1, pp. 14-16, 2008. 


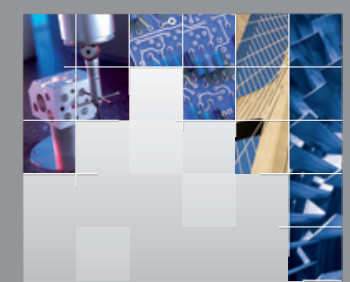

\section{Enfincering}
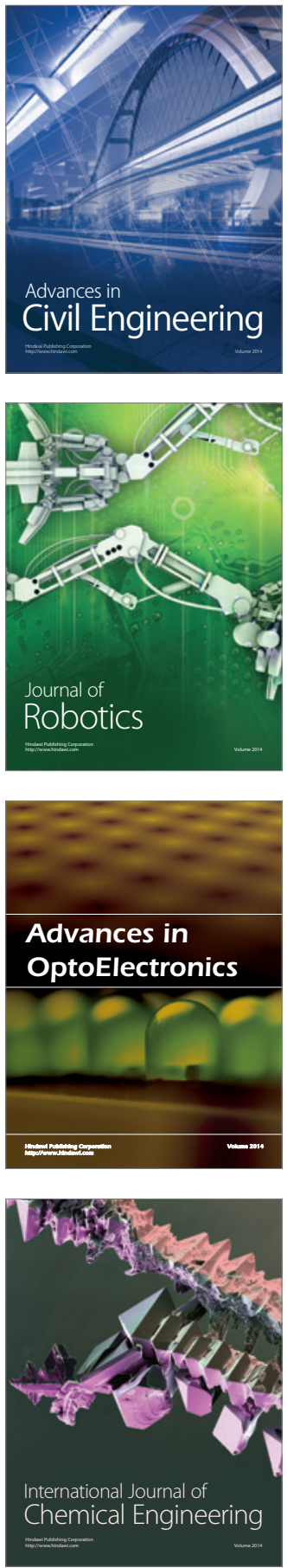

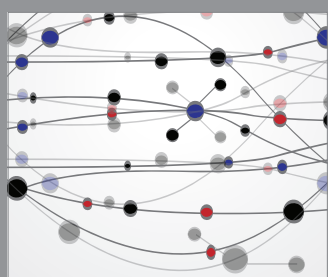

The Scientific World Journal

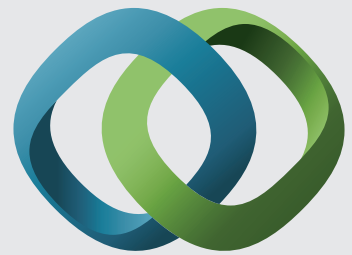

\section{Hindawi}

Submit your manuscripts at

http://www.hindawi.com
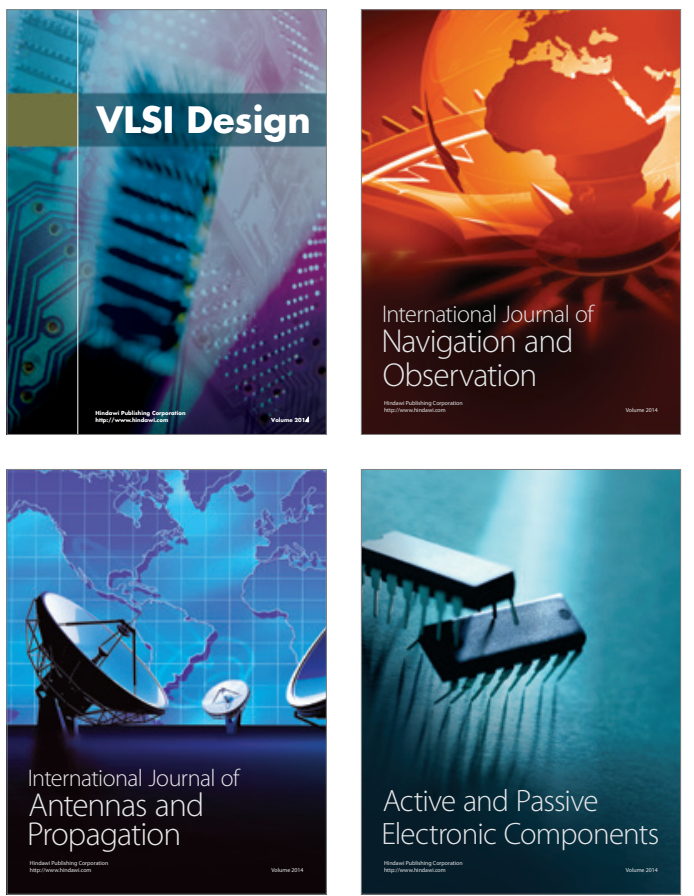
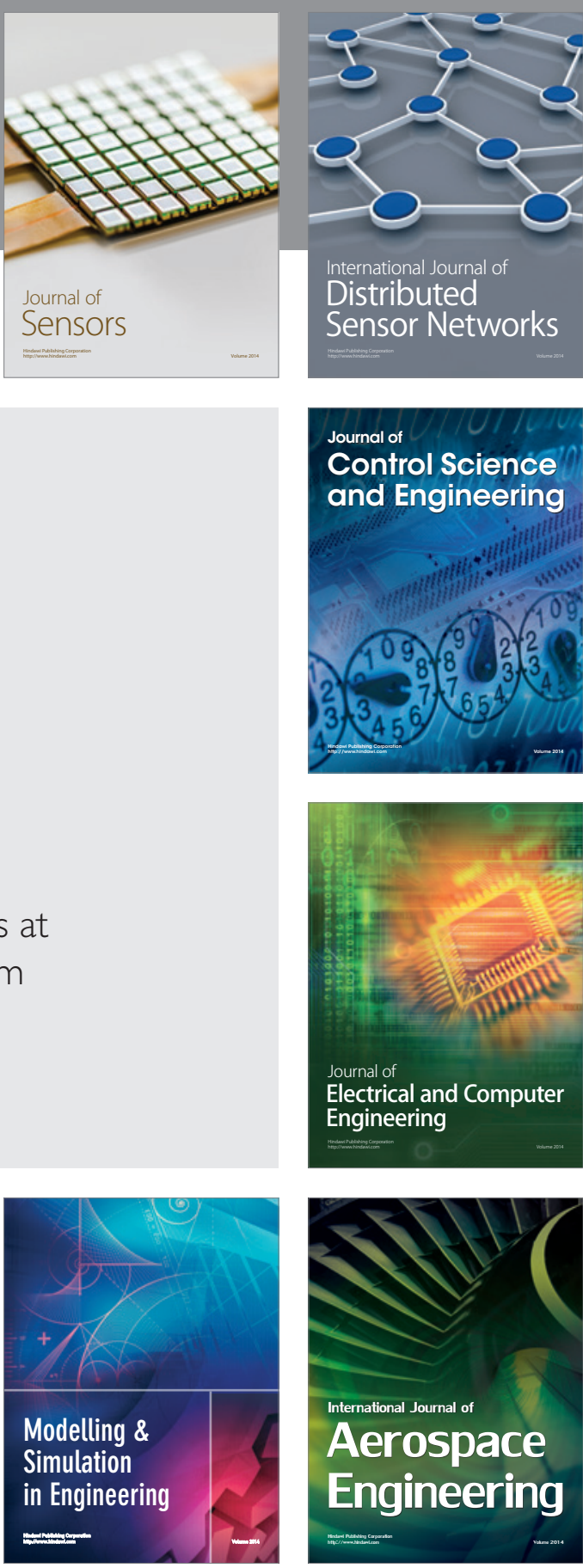

International Journal of

Distributed

Sensor Networks

Journal of

Control Science

and Engineering
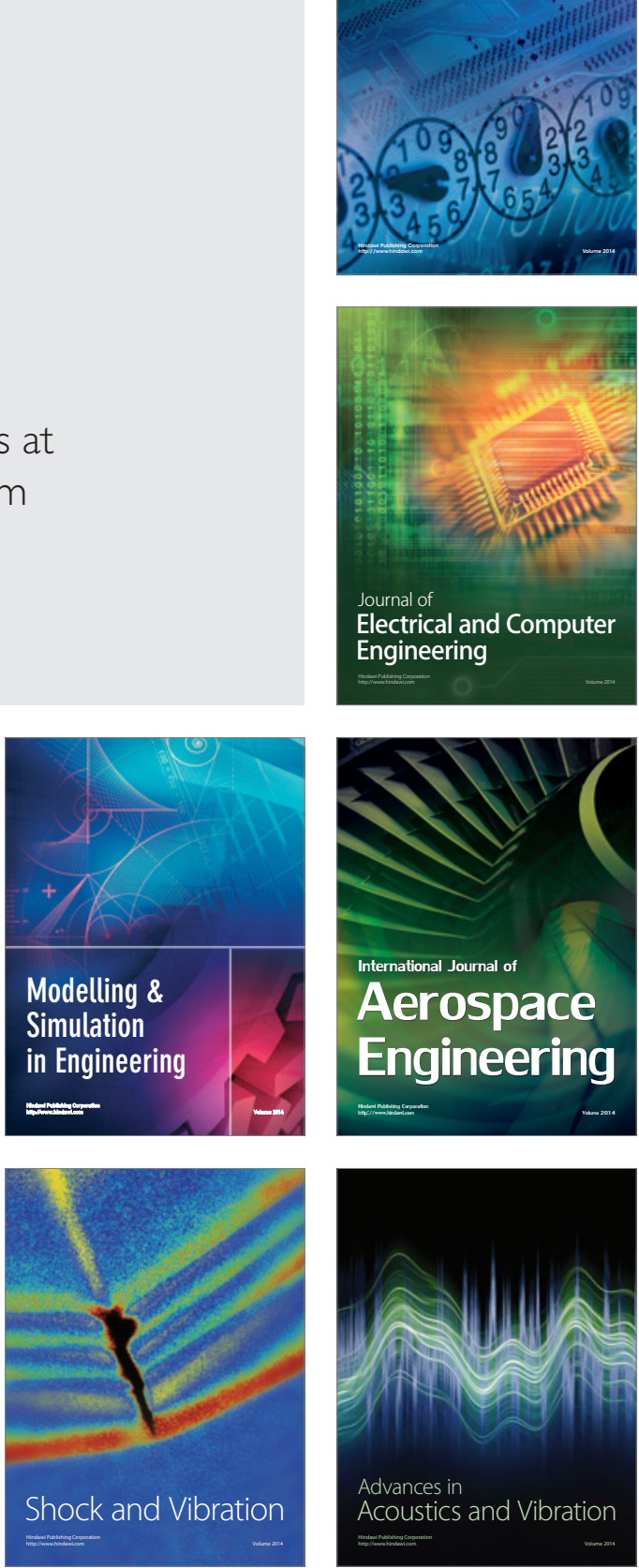